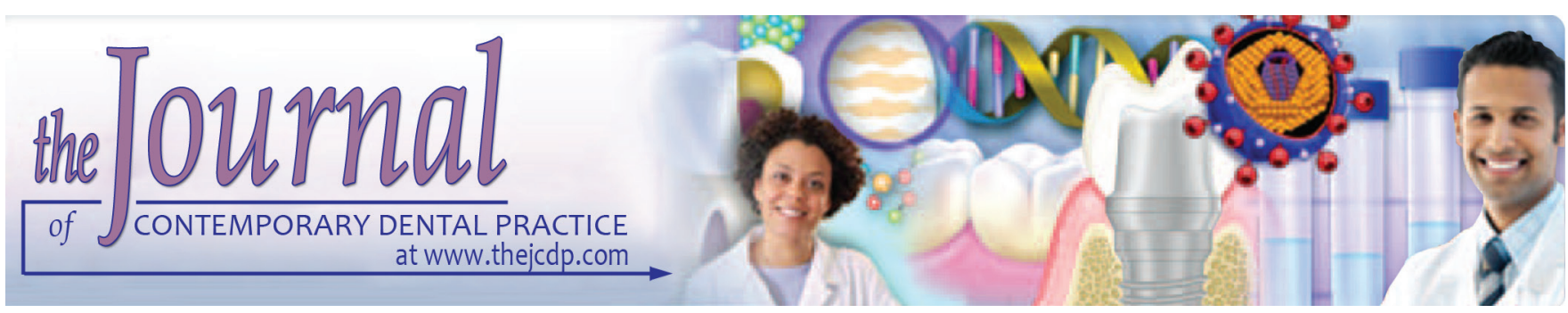

\title{
Role of E-cadherin in Progression of Oral Squamous Cell Carcinoma: A Retrospective Immunohistochemical Study
}

\author{
${ }^{1}$ Awadhesh Gupta, ${ }^{2}$ Seema Sharma, ${ }^{3}$ Monisha Batra, ${ }^{4}$ Mohammed Abidullah, ${ }^{5}$ Swathi Bhuvinder, ${ }^{6}$ Padmavathi Katragadda
}

\section{ABSTRACT}

Aim: The aim of present study was to assess the role of E-cadherin in oral carcinogenesis by comparing their expressions in normal oral mucosa, oral epithelial dysplasia (OED), and oral squamous cell carcinoma (OSCC).

Materials and methods: To elucidate the pattern of E-cadherin expression in oral carcinogenesis, 21 archival cases of formalinfixed paraffin-embedded sections of OSCC, 21 OED, and 7 normal oral mucosa samples as control were used for the study.

Results: We observed reduction/loss of E-cadherin in membranous expression pattern and staining intensity with progression from dysplasia to oral cancer.

Conclusion: A decrease in staining intensity and loss of E-cadherin membranous expression were noted from dysplasia to carcinoma, suggesting its role as a tumor suppressor gene.

Clinical significance: E-cadherin can be used as a biomarker to assess and evaluate the progression and prognosis of oral dysplastic lesions and OSCC.

Keywords: Cell adhesion molecules, Dysplastic epithelium, E-cadherin, Immunohistochemistry, Tumor markers.

${ }^{1}$ Department of Oral and Maxillofacial Pathology, ESIC Dental College and Hospital, Rohini, New Delhi, India

${ }^{2}$ Department of Oral and Maxillofacial Pathology and Microbiology, Santosh Dental College and Hospital, Ghaziabad Uttar Pradesh, India

${ }^{3}$ Private Clinical Practitioner, Chanakya, New Delhi, India

${ }^{4}$ Department of Oral Pathology and Microbiology, Pacific Academy of Higher Education \& Research Society, PAHER University, Udaipur, Rajasthan, India

${ }^{5}$ Department of Paediatrics and Preventive Dentistry, Malla Reddy Dental College for Women, Hyderabad, Telangana, India

${ }^{6}$ Department of Periodontics, Malla Reddy Institute of Dental Sciences, Hyderabad, Telangana, India

Corresponding Author: Awadhesh Gupta, Department of Oral and Maxillofacial Pathology, ESIC Dental College and Hospital Rohini, New Delhi, India, Phone: +919711927559, e-mail: drawadheshgupta@gmail.com
How to cite this article: Gupta A, Sharma S, Batra M, Abidullah M, Bhuvinder S, Katragadda P. Role of E-cadherin in Progression of Oral Squamous Cell Carcinoma: A Retrospective Immunohistochemical Study. J Contemp Dent Pract 2018;19(9):1105-1110.

\section{Source of support: Nil}

Conflict of interest: None

\section{INTRODUCTION}

Cell-cell adhesion plays a fundamental and dynamic role in the development and maintenance of multicellular organisms as well as overall tissue organization and functions. ${ }^{1}$ Cadherins are a family of cell-cell adhesion molecules with an important role in intercellular adhesion. ${ }^{2}$ They are cell surface membrane proteins that mediate homophilic $\mathrm{Ca}++-$ dependent cell-cell adhesion which is crucial for structural organization and differentiation of cells. ${ }^{3}$ Cadherins constitute a superfamily and the original cadherins are now called as "classical cadherins." Approximately 20 members of cadherins are included in the classic cadherin family depending on their domain structures.

E-cadherin is a $120 \mathrm{kDa}$ transmembrane glycoprotein of type-I cadherin super family. It belongs to the family of cell-cell adhesion molecules essential for maintaining the structural integrity and organization of stratified squamous epithelium. ${ }^{1,3}$ It is encoded by the $\mathrm{CDH} 1$ gene that is located on chromosome $16 \mathrm{q}-22.1$ and is a classical cadherin that forms the key functional component of adherence junctions between epithelial cells. ${ }^{3}$

In normal oral epithelium, E-cadherin is expressed in the lower spinous layers and basal cell layer, but not at the stromal surface of basal cells. ${ }^{4}$ E-cadherin plays a key role in junctional adherence through binding of the extracellular domain of E-cadherin molecules of adjacent cells. Recent studies implicate that cadherins regulate interaction of growth factor receptors with ligands and modulate 
their signaling. ${ }^{4,5}$ Cadherins regulate the growth factor signaling by recruiting the receptors at the cell surface, stimulating the receptor dimerization and modulating their activities.

Dysplastic oral epithelial lesions may be morphological phenotypes of the different steps in the progression from normal to malignant tissue. Traditionally, OED has been considered to be the progenitor for malignant change. ${ }^{6}$ There is reduction in E-cadherin expression in OED as severity of dysplasia is increased from mild to severe.

Carcinogenesis is a multistage process which requires modulation of cell-cell and cell-stromal interactions. ${ }^{7}$ Loss of E-cadherin expression has been noted with poorly differentiated morphology in a large number of malignancies, such as cervix and esophagus including head and neck. ${ }^{1}$ Development of malignant tumors, in particular, the transition from benign lesion to invasive metastatic cancer is characterized by a tumor cell's ability to overcome cell-cell adhesion and to invade the surrounding tissues. $^{8}$

\section{MATERIALS AND METHODS}

The tissue specimen for the study comprised a total of 49 formalin-fixed and paraffin-embedded tissue blocks retrieved from the Department of Oral and Maxillofacial Pathology and Microbiology, Santosh Dental College and Hospital, Santosh University, Ghaziabad, New Delhi, India. The archival material comprised 21 cases each of oral dysplastic epithelium and OSCC and 7 cases of normal oral mucosa as control.

For the purpose of this study, samples were distributed into three different groups: Group I included seven samples each of histopathologically confirmed cases of mild, moderate, and severe epithelial dysplasia, group II included seven samples each of histopathologically confirmed cases of well, moderately, and poorly differentiated squamous cell carcinoma (SCC), and group III included seven cases of histologically confirmed cases of normal oral mucosa (as control group). The samples of epithelial dysplasia were graded as per World Health Organization (WHO) 2005 classification as mild, moderate, and severe. ${ }^{7}$ Similarly, the samples of SCC were graded as per Broder's criteria as well-differentiated, moderately differentiated, and poorly differentiated SCC. ${ }^{10}$

The staining intensity was assessed according to Zhang et al and membranous expression pattern of E-cadherin was assessed according to Bankfalvi A et al. ${ }^{11,12}$ Intensity of staining according to Zhang et al grading system: (a) $0 /$-ve = absent/loss of expression; (b) $1(+)=$ weak/light staining; (c) $2(++)=$ moderate staining; (d) $3(+++)=$ strong/intense staining. Membranous staining expression pattern according to Bankfalvi A et al: $0=0$ to $10 \%$ of cells showed a complete cellular membranous staining; $1=11$ to $50 \%$ of cells showed a complete cellular membranous staining; $2=51$ to $75 \%$ of cells showed a complete cellular membranous staining; $3=76$ to $100 \%$ of cells showed a complete cellular membranous staining.

The formalin-fixed paraffin-embedded tissue blocks of normal oral mucosa, epithelial dysplasia, and SCC were selected. Two sections each of $3 \mu \mathrm{m}$ thickness were cut using a semi-rotary microtome and the sections were stained by hematoxylin and eosin. The serial sections of the same were stained with E-cadherin immunohistochemical reagent using avidin-biotin complex method. The expression of E-cadherin was analyzed in the entire length of the epithelium in normal, dysplastic, and stratified SCC epithelium, and evaluated by two independent observers using light microscope.

Areas representing the epithelium were analyzed in high power to obtain a clear and precise count of the labeled cells; 3 to 4 representative fields at 400 magnifications (40×) were selected and 100 E-cadherin positive cells were counted out in the representative fields. Thus, by selecting random areas which were not in continuum with each other, a possible error in recounting the same cells was minimized. The images of each of the selected fields were obtained with a binocular light microscope $(40 \times)$, and then transferred to a computer system for analysis which was done by using a grid. Intensity of membranous staining was assessed by two independent observers using light microscope ( $\times 400$ original magnifications).

\section{STATISTICAL ANALYSIS}

The software used for the statistical analysis was Statistical Package for the Social Sciences version 21.0 and Epi Info version 3.0. Chi-square test was used to test whether the expression of E-cadherin was statistically significant. A p-value $<0.05$ was considered statistically significant.

\section{OBSERVATION AND RESULTS}

In the present study, all the control sections of normal oral mucosa strongly expressed E-cadherin on their cell membrane. A strong membranous homogenous staining of E-cadherin was seen on the cells of the basal, parabasal, and spinous layers. The superficial layers of the epithelium and the portion of the basal cells toward the basement membrane did not yield an E-cadherin expression (Fig. 1A). Out of 21 cases of OED, E-cadherin immunoreactivity was moderately positive in $38 \%$ cases, strong positive in $38 \%$ cases, while negative or weak immunoreactivity was not observed in cases of OED (Tables 1 and 2). The reduced expression 
Role of E-cadherin in Progression of Oral Squamous Cell Carcinoma

\begin{tabular}{lllll}
\hline \multicolumn{4}{c}{$\begin{array}{c}\text { Table 1: E-cadherin staining intensity } \\
\text { among different grades of OED }\end{array}$} \\
\cline { 2 - 5 } Grades of dysplasia & Absent & Light & Moderate & Intense \\
\hline Mild dysplasia $(\mathrm{n}=7)$ & 0 & 0 & 1 & 6 \\
Moderate dysplasia $(\mathrm{n}=7)$ & 0 & 0 & 5 & 2 \\
Severe dysplasia $(\mathrm{n}=7)$ & 0 & 5 & 2 & 0 \\
\hline
\end{tabular}

Table 2: E-cadherin membranous expression among different grades of OED

\begin{tabular}{lllll}
\hline & \multicolumn{5}{c}{ E-cadherin membranous expression } \\
\cline { 2 - 5 } Grades of dysplasia & 0 & $1(+)$ & $2(++)$ & $3(+++)$ \\
\hline Mild dysplasia $(\mathrm{n}=7)$ & 0 & 0 & 1 & 6 \\
Moderate dysplasia $(\mathrm{n}=7)$ & 0 & 0 & 4 & 3 \\
Severe dysplasia $(\mathrm{n}=7)$ & 0 & 0 & 6 & 1 \\
\hline
\end{tabular}
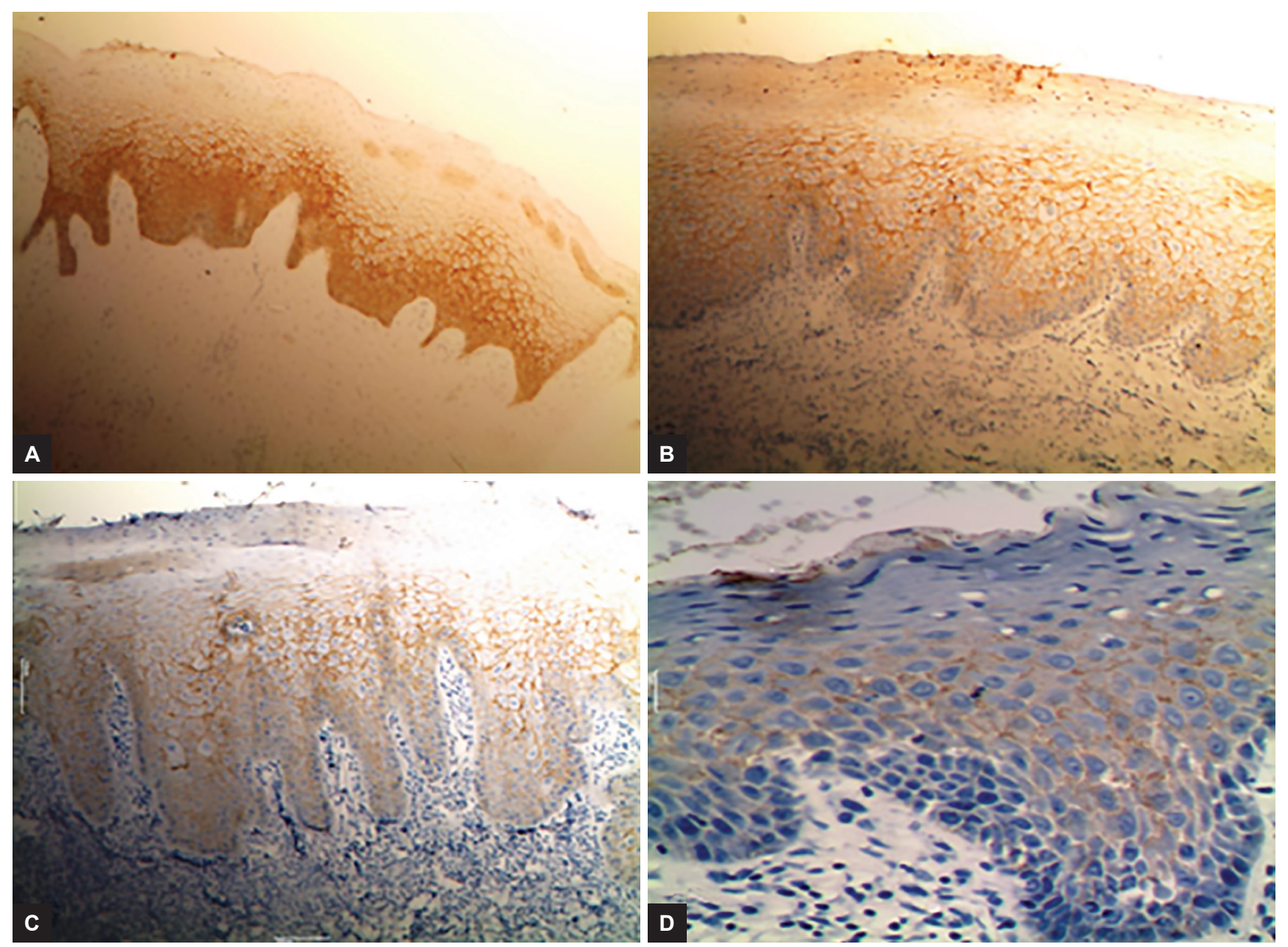

Figs 1A to D: (A) Normal oral mucosa showing all the cells of basal, parabasal, and superficial spinous layers of the epithelium exhibiting strong intensity and complete membranous expression of E-cadherin (IHC; 10x); (B) mild dysplasia showing all the cells of basal, parabasal, and superficial spinous layers of the epithelium exhibiting strong intensity and complete membranous expression of E-cadherin (IHC; 10×); (C) moderate dysplasia showing few basal cells and all the cells of parabasal and superficial spinous layers of the epithelium exhibiting strong intensity and complete membranous expression of E-cadherin (IHC; 10x); (D) severe dysplasia showing few basal and parabasal cells and all the cells of superficial spinous layers of the epithelium exhibiting strong intensity and reduced membranous expression of E-cadherin (IHC; 40x)

of E-cadherin was observed from mild to severe degree of dysplasia, suggesting the negative correlation with E-cadherin expression (Figs 1B to D).

In the tissues of OSCC, 10 tissues showed moderateto-weak intensity in all the cell layers. In the remaining 11 cases, the basal cells were negative for E-cadherin. The number of cells exhibited complete membranous pattern of E-cadherin immunostaining was variable (Tables 3 and 4). Variation in the expression of E-cadherin was found between the different layers in different groups of OSCC in terms of both intensity and membranous staining. The chi-square values obtained in the cases of normal mucosa and well-differentiated SCC with regard to both intensity and expression pattern were significant ( $p$-value $=0.018$ and $p=0.027$ ). Among the moderately differentiated SCC cases and poorly differentiated SCC cases, both intensity and expression pattern were significant. The chi-square values obtained in the cases of OED and OSCC in terms of staining intensity and membranous expression were also significant (Tables 3 and 4). 
Table 3: E-cadherin staining intensity among different groups of OSCC

\begin{tabular}{lllll}
\hline & \multicolumn{4}{c}{ E-cadherin staining intensity } \\
\cline { 2 - 5 } Differentiation of OSCC & Absent & Light & Moderate & Intense \\
\hline WDSCC $(n=7)$ & 0 & 4 & 2 & 1 \\
MDSCC $(n=7)$ & 4 & 2 & 1 & 0 \\
PDSCC $(n=7)$ & 7 & 0 & 0 & 0
\end{tabular}

WDSCC: Well-differentiated SCC; MDSCC: Moderately differentiated SCC; PDSCC: Poorly differentiated SCC

\section{DISCUSSION}

The expression of E-cadherin was evaluated through different grades of OED and OSCC. The normal oral epithelium showed intense and complete cellular membranous staining of E-cadherin in the basal, parabasal, and lower parts of spinous layer. The staining was absent in the superficial layers including the uppermost layer (Fig. 1A). Similar observations were also made by various researchers including Wheelock et $\mathrm{al}^{13}{ }^{13}$ Williams et al, ${ }^{14}$ and Kaur et al. ${ }^{15}$ In normal oral epithelium, membranous E-cadherin staining was noticed to be localized at the intercellular junctions and lost at the upper third of the epithelium; this may be attributed to the normal desquamation process (Fig. 1A). In the present study, a controlled absence of E-cadherin staining was observed at the stromal surface of basal cells. The above observation supports that E-cadherin is inhibited until a cell replicates and is present in the cells that have divided and differentiated. This forms the basis of the hypothesis that E-cadherin is involved in the maintenance of polarity and tissue architecture. ${ }^{2}$

Oral epithelial dysplasias have not been commonly explored for E-cadherin expression. Therefore, there has been less consistency in results of previous studies. ${ }^{1,7,14,15}$ In the present study, out of 21 cases of OED, the expression pattern was similar to normal mucosa in all mild dysplasias with strong membranous staining with absence of staining in the superficial layers (Fig. 1B). Slight loss of expression was noted as severity of dysplasia increased from moderate to severe dysplasia (Figs $1 C$ and D and Table 2).

This observation is in alignment with the previous studies performed by Yuwanati et al, ${ }_{1}^{1}$ Califano et $\mathrm{al}_{1}{ }^{16}$ and Santos-García et al. ${ }^{17}$ E-cadherin expression in mild epithelial dysplasia was present in suprabasal and basal cell layers similar to normal oral epithelium. In moderate epithelial dysplasia, E-cadherin expression was present in suprabasal, but reduced in the basal cell layers. The reduction in the expression of E-cadherin was noted in terms of intensity and number of cells exhibiting complete cellular membranous staining, especially in the basal and parabasal layers. This observation is in accordance with the studies performed by Williams et $\mathrm{al}^{14}$
Table 4: E-cadherin membranous expression among different groups of OSCC

\begin{tabular}{lllll}
\hline & \multicolumn{5}{c}{ E-cadherin Membranous Expression } \\
\cline { 2 - 5 } Differentiation of OSCC & 0 & $1(+)$ & $2(++)$ & $3(+++)$ \\
\hline $\operatorname{WDSCC}(\mathrm{n}=7)$ & 0 & 2 & 4 & 1 \\
$\operatorname{MDSCC}(\mathrm{n}=7)$ & 4 & 1 & 2 & 0 \\
PDSCC $(\mathrm{n}=7)$ & 7 & 0 & 0 & 0 \\
\hline
\end{tabular}

WDSCC: Well-differentiated SCC; MDSCC: Moderately differentiated SCC; PDSCC: Poorly differentiated SCC

and Santos-Garcia et al. ${ }^{17}$ Their studies also showed reduction/loss of E-cadherin expression with increasing severity of dysplasia. The reduction in E-cadherin expression becomes more evident in carcinoma in situ and microinvasive carcinoma cases. Hence, the present study is consistent with Yuwanati et al, ${ }^{1}$ Yogesh et al, ${ }^{7}$ and Mehendiratta et $\mathrm{al}^{18}{ }^{18}$ and suggests that these alterations in E-cadherin expression are the result of the progression of dysplasia and could be an early event in the process of oral carcinogenesis.

Among the 21 cases of OSCC, one well-differentiated SCC showed strong membranous expression pattern of E-cadherin similar to that of normal stratified squamous epithelium (Figs 2A and B and Table 2). Among the moderately differentiated OSCC, four cases showed loss in expression (Fig. 3A), one case showed weak expression, and two cases showed moderate expression of E-cadherin (Fig. 3B). None of these were completely negative for E-cadherin expression. Moderately differentiated OSCC showed heterogeneous staining, where the central cell mass of tumor islands was differentiated and expressed membranous E-cadherin staining, while the cells at the periphery were dedifferentiated and expressed cytoplasmic expression only (Fig. 3B). Among the poorly differentiated SCC, all the seven cases showed loss of membranous expression of E-cadherin (Table 1). There was a significant difference in staining intensity and staining location in poorly differentiated OSCC when compared with well-differentiated SCC and moderately differentiated SCC (Table 3). Poorly differentiated OSCC showed sheets of tumor cells with complete loss of membranous E-cadherin expression.

The present study showed consistent results where the loss of E-cadherin expression was associated with the histological grade of differentiation of OSCC. Thus, our study is in accordance with the studies done by Williams et $\mathrm{al}_{1}{ }^{14}$ Hung et $\mathrm{al},{ }^{19}$ Sorcher et $\mathrm{al}_{,}{ }^{20}$ and Mehendiratta et al. ${ }^{18}$ They also showed an inverse correlation of E-cadherin expression with the histological grades of differentiation. It is now well understood that E-cadherin regulates growth factor signaling by recruiting the receptor at the cell surface, stimulating the receptor dimerization, and modulating their activity. ${ }^{5}$ In human tumors, the loss of E-cadherin-mediated cell 


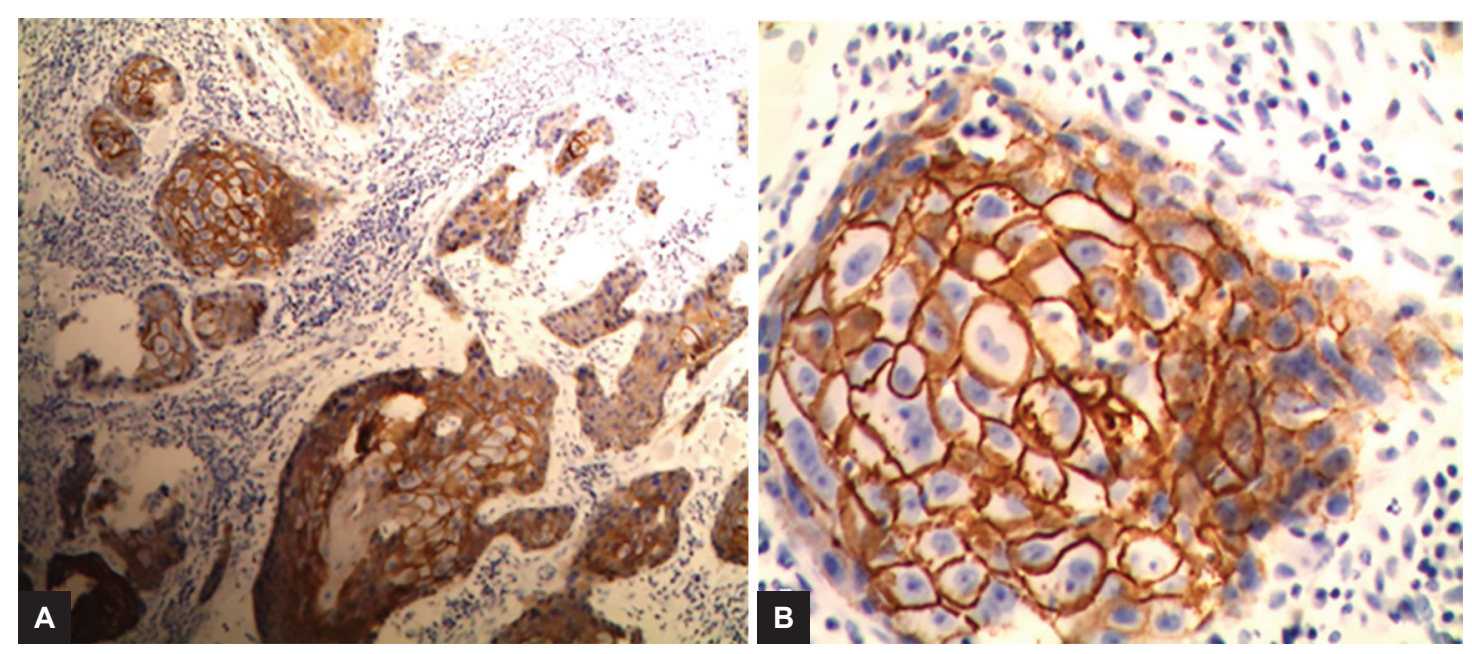

Figs 2A and B: Well-differentiated OSCC. (A) All the cells of the tumor island exhibit strong intensity and complete membranous expression of E-cadherin (IHC; 10x). (B) All the cells of the tumor island exhibit strong intensity and complete membranous expression of E-cadherin (IHC; 40x)
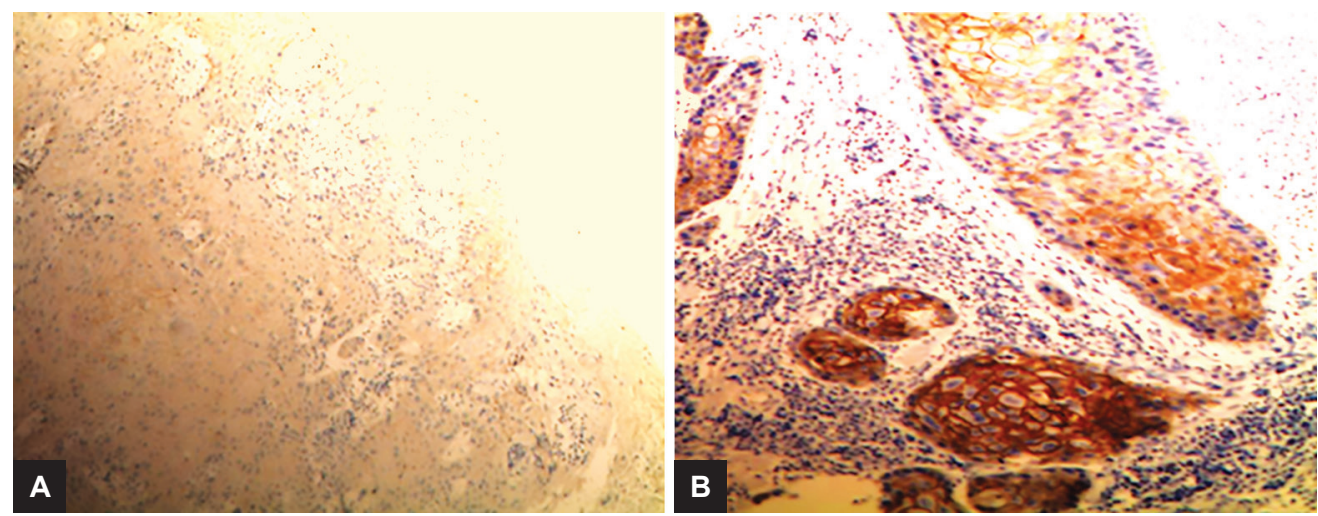

Figs 3A and B: Moderately differentiated OSCC. (A) Most of the tumor cells exhibit strong-moderate intensity and membranous expression while few cells exhibit reduced expression of E-cadherin (IHC; 10x). (B) Some of the tumor cells exhibit heterogeneous staining, where the central mass of tumor island was differentiated and expressed membranous E-cadherin staining, while the cells at the periphery were dedifferentiated and expressed cytoplasmic expression only (IHC; 10x)

adhesion correlates with the loss of epithelial morphology and with the acquisition of metastatic potential by the neoplastic cells.

In the present study, we demonstrated a progressive reduction in the number of cells exhibiting complete cellular membranous expression pattern of staining and intensity with increasing severity of dysplasia to cancer. We also investigated the loss of expression pattern or reduction of E-cadherin staining semi-quantitatively which appeared to begin from the basal cell layer to superficial layers. This alternation is the result of dysplasia toward the cell phenotype with the ability to invade..$^{21}$

Proliferation of epithelial cells expands into the superficial layers with severity, which leads to the loss of E-cadherin expression in the basal cell layers. The loss of E-cadherin expression in the spinous layers could be due to lesser number of cells reaching the stage of differentiation. Hence, our study confirms the previous studies that the loss of E-cadherin expression is an early event in the evolution and progression of carcinomas. From the present study, we found that premalignant lesions with the loss of E-cadherin expression are at the greater risk in terms of malignant transformation from premalignancy to cancer.

\section{CONCLUSION}

E-cadherin plays an important role in the process of oral carcinogenesis by its reduced expression in OED and OSCC. It also suggests that E-cadherin is an "invasion suppressor" molecule and loss of E-cadherin during carcinogenesis enhances the invasion of adjacent normal tissues. Additional studies on larger sample are needed to investigate the mechanism by which the reduction and loss of E-cadherin expression during malignant transformation occur. Thus, E-cadherin is known to be a tumor invasion suppressor gene and plays a central role in oral cancers. 


\section{Clinical Significance}

Loss of E-cadherin expression pattern and reduction in intensity can be used as a reliable marker for progression of potentially malignant disorders toward invasive malignancy. It may also aid in providing new strategies for early diagnosis, prevention, and treatment planning of oral cancer to bring down the mortality rate.

\section{REFERENCES}

1. Yuwanati MB, TupkariJV, Avadhani A. Expression of E-cadherin in oral epithelial dysplasia and oral squamous cell carcinoma: an in vivo study. J Clin Exp Invest 2011 Dec;2(4):347-353.

2. Imai K, Maeda G, Chiba T. Cadherin expression and progression of squamous cell carcinoma of the oral cavity. In: Li X, editor. Squamous cell carcinoma. Croatia: InTech; 2012.

3. Kantak SS, Kramer RH. E-cadherin regulates anchorageindependent growth and survival in oral squamous cell carcinoma cells. J Biol Chem 1998 Jul;273(27):16953-16961.

4. Diniz-Freitas M, Garcia-Caballero T, Antunez-Lopez J, Gandara-Rey JM. Loss of expression of E-cadherin correlates with increased invasiveness, metastasis and unfavourable prognosis. Oral Oncol 2006;49:190-200.

5. Cavallaro U, Dejana E. Adhesion molecules signaling: not always a sticky business. Nat Rev Mol Cell Biol 2011 Mar;12(3):189-197.

6. von Zeidler SV, de Souza Botelho T, Mendonca EF, Batista AC. E-cadherin as a potential biomarker of malignant transformation in oral leukoplakia: a retrospective cohort study. BMC Cancer 2014 Dec;14:972.

7. Yogesh T, Narayan T, Shreedhar B, Shashidara R, Leekymohanty. The expression of E-cadherin and cathepsin$\mathrm{D}$ in normal oral mucosa, oral epithelial dysplasia and oral squamous cell carcinoma: a comparative analysis between immunohistochemistry and routine histo- pathology. J Oral Maxillofac Pathol 2011 Sep;15(3):288-294.

8. Christofori G. Semb H. The role of cell-adhesion molecule E-cadherin as a tumour-suppressor gene. Trends Biochem Sci 1999 Feb;24(2):73-76.

9. Branes L, Eveson JW, Reichert P, Sidransky D. Tumours of the oral cavity and oropharynx. Pathol Genet 2005;67: 177-179.
10. Doshi Neena P, Shah Siddharth A, Patel Keyuri B, Jhabuawala Munira F. Histological grading of oral cancer: a comparison of different systems and their relation to lymph node metastasis. Natl J Community Med 2011;2(1):136-143.

11. Zhang W, Alt-Holland A, Margulia A, Shamis Y, Fusenig NE, Rodeck U, Garlick JA: E-cadherin loss promotes the initiation of squamous cell carcinoma invasion through modulation of integrin-mediated adhesion. J Cell Sci 2006;119:283-291.

12. Bankfalvi A, Krassort M, Vegh A, Felszeghy E, Piffko J. Deranged expression of the E-cadherin/beta-catenin complex and the epidermal growth factor receptor in the clinical evolution and progression of oral squamous cell carcinomas. J Oral Pathol Med 2002 Sep;31(8):450-457.

13. Wheelock MJ, Shintani Y, Maeda M, Fukumoto Y, Johnson KR. Cadherin switching. J Cell Sci 2008 Mar;121(Pt 6):727-732.

14. Williams HK, Sanders DS, Jankowski JA, Landini G, Brown AM. Expression of cadherins and catenins in oral epithelial dysplasia and squamous cell carcinoma. J Oral Pathol Med 1998 Aug;27(7):308-317.

15. Kaur G, Carnelio S, Rao N, Rao L. Expression of E-cadherin in primary oral squamous cell carcinoma and metastatic lymph nodes: an immunohistochemical study. Indian J Dent Res 2009 Jan-Mar;20(1):71-76.

16. Califano J, van der Riet P, Westra W, Nawroz H, Clayman G, PiantadosiS, Corio R, Lee D, Greenberg B, Koch W, et al. Genetic progression model for head and neck cancer: implications for field cancerization. Cancer Res 1996 Jun;56(11):2488-2492.

17. Santos-García A, Abad-Hernández MM, Fonseca-Sánchez E, Julián-González R, Galindo-Villardón P, Cruz-Hernández JJ, Bullón-Sopelana A. E-cadherin, laminin and collagen IV expression in the evolution from dysplasia to oral squamous cell carcinoma. Med Oral Pathol Cir Buccal 2006 Mar;11(2): E100-E105.

18. MehendirattaM,SolomonMC, BoazK, Guddattu V,MohindraA Clinico-pathological correlation of E-cadherin expression at the invasive tumor front of Indian oral squamous cell carcinomas: an immunohistochemical study. 2014 May;18(2):217-222.

19. Hung KF, Chang CS, Liu CJ, Lui MT, Cheng CY, Kao SY. Differential expression of E-expression in metastatic lesions comparing to primary oral squamous cell carcinoma. J Oral Pathol Med 2006 Nov;35(10):589-594.

20. Sorcher SM, Russack V, Cagle M, Feramisco JR, Green MR. Immunolocalization of E-cadherin in human head and neck cancer. Arch Pathol Lab Med 1995 Jan;119(1):82-84. 